\title{
Die Loreley, Germania und Die Wacht am Rhein
}

\author{
Ernst-Ullrich Pinkert \\ (Ålborg-Universitæt)
}

Mehr als 65 Jahre lang setzte sich der Schriftsteller und Literaturkritiker Rudolf Gottschall (18231909) mit Heine auseinander - in Aufsätzen, Rezensionen, Literaturgeschichtsbüchern und sogar in Gedichten. Gottschall war mit Heine persönlich bekannt: er hatte ihn 1851 in Paris besuchtund ging in seinen Memoiren davon aus, daß Heine "günstig über [ihn] dachte". (1) Auch wenn Gottschall von Heines 'Charakter' mitunter enttäuscht war, so blieb er doch stets ein großer Verehrer von Heines 'Talent'. (ㅁ) Daran änderte sich auch nichts, als aus dem liberalen Oppositionellen der 1840er Jahre ein Geheimer Hofrat mit Adelstitel geworden war, der Hymnen auf den deutschen Kaiser verfasste. (므) - Der alte Gottschall nahm Heine sogar gegen Angriffe von rechts in Schutz:

\begin{abstract}
Als "vaterlandsloser Geselle" von einem Teil unseres patriotischen Bürgertums gebrandmarkt, in literarischen Fehden oft mit Erbitterung befehdet, behauptet er, bei unparteiischer Würdigung, eine hervorragende Stellung in unserer Literatur als eines ihrer größten lyrischen Talente. ()
\end{abstract}

Von seinem ersten Buch (므) bis zu seinem letzten (ㅁ) - von 1842 bis 1908/09 - hat sich Gottschall immer wieder mit Heines Loreley ( $\underline{7})$ und der Loreley-Thematik auseinandergesetzt - als Literaturhistoriker/ -kritiker und als Dichter. Die Verschiebungen und Brüche, die sich in Gottschalls Loreley-Überlegungen feststellen lassen, sind zugleich Indizien für ideologische Verschiebungen und Brüche in der Entwicklung großer Teile des liberalen Bürgertums im 19. Jahrhundert, dessen Weg von der Vormärz-Opposition über die verpatzte Revolution von 1848 in die "machtgeschützte Innerlichkeit" (Thomas Mann) des Kaisertums führte. In diesen Jahren verwandelt sich im öffentlichen Diskurs die kosmopolitische Freiheitsgöttin Germania in eine deutsche Siegesgöttin; gleichzeitig gibt es in Deutschland mancherlei Versuche, die LoreleyAllegorie in das Zeichensystem des Kaiserreichs hineinzupressen. Auch der Dichter Gottschall unternimmt (um 1883) einen solchen Versuch.

In seinen literaturtheoretischen und -historischen Werken verwendet Gottschall Heines Loreley als Demonstrationsobjekt und Beweisstück; (ㅁ) 1868 z.B. erwähnt er "das unvergänglich schöne 'Loreleilied'", um "den reinen und harmonischen Charakter mancher Heine'schen Gedichte gegenüber einer einseitigen Betrachtung außer Zweifel zu stellen". (ㅁ) Eine zentrale Rolle spielt die Loreley jedoch in Gottschalls Buch Die deutsche Nationalliteratur des neunzehnten Jahrhunderts. (10) Wiederholt hatte Gottschall es als das Hauptverdienst von Heines "Liedern" angesehen, die "Auflösung der Romantik" betrieben und "die Ära der modernen deutschen Dichtung" eingeleitet zu haben. (11) In seiner Nationalliteratur begründet er dieses Urteil unter ausdrücklicher Bezugnahme auf die Loreley und erkennt dabei diesem Gedicht eine Schlüsselrolle bei der Entwicklung der modernen deutschen Literatur zu.

Gottschall schätzt Heines "Anmuth, Empfindung, Naturandacht, Gedankenschwung" und weiß, daß das "Liebliche und Träumerische" von Heines Lyrik hier seinen Ursprung hat; dennoch mißt er diesen positiven Eigenschaften keine "originale Bedeutung" bei. Neu sei lediglich Heines "diabolische Kraft" gewesen, "welche jedes Gefühl, jeden Gedanken wieder zertrümmert, welche das Schöne nur als Torso kennt". In seinem Bemühen, diese "diabolische Kraft" dingfest zu 
machen, verweist er auf die Loreley:

Es gehörte die Meisterschaft in lieblichen und süßen Klängen dazu, um die

Vernichtung und Versöhnung derselben umso greller und empfindlicher zu machen.

Die süße Lorelei, die den Schiffer verlockt, berauscht und dann in's Verderben stürzt, die Sphinx, die mit dem Göttermunde küßt und dabei mit den Tatzen zerfleischt, sind die Lieblings-Vignetten seiner Dichtungen. (12)

Die Loreley ist für Gottschall der Inbegriff von Heines Lyrik, weil hier sowohl die Auflösung der Romantik als auch der Anbruch der Moderne stattfinde; und doch interpretiert er die "Dichtweise" der Loreley als "poetische Verhöhnung der Poesie" und spricht ihr "die absolute Berechtigung" ab. Nur in einer Zeit des Übergangs könne diese "Dichtweise" noch eine gewisse Rolle spielen, denn:
Was Heine's Muse auflöst, ist nicht die echte, ewige, organisch schaffende Poesie; es ist die verzückte, traumhafte, empfindungskranke Romantik. Er vergiftet nur eine
Schwindsüchtige! Was aber reif ist für den Tod, das mag in's Grab sinken und Raum geben einer neuen und gesunden Welt. Auch diese Gesundheit kündigt sich vielfach bei Heine an, doch nur als Postulat.

Es ist bemerkenswert, daß der Literaturtheoretiker Gottschall, der in seinem von Arnold Ruge und Friedrich Theodor Vischer beeinflußten ästhetischen Koordinatensystem kategorisch zwischen 'krank' und 'gesund' unterscheidet, jenes Gedicht, dem er selbst "Unvergänglichkeit" bescheinigt hat, keineswegs als Ausdruck des Gesunden gelten lassen will. Vor diesem Hintergrund ist die Auseinandersetzung mit den beiden Texten, in denen sich der Dichter Gottschall mit der LoreleyThematik beschäftigt, besonders interessant.

In beiden Texten - in dem frühen Gedicht Dem Rhein (1842) (13) und in dem späten Am Rhein (14) (siehe Textabdruck) - knüpft Gottschall implizit an die Allegorie-Tradition an, die sich im Zuge der Rezeption von Heines Loreley entwickelt hatte. (15) Doch in der Bewertung dieser Tradition unterscheiden sich die beiden Gedichte. Das frühe schließt nahtlos an sie an, das späte aber relativiert sie, denn hier wird neben einer 'blassen' Loreley, die im wahrsten Sinne des Wortes sangund klanglos von der Bildfläche verschwinden will, eine monumentale Germania als 'Tat'-kräftiger Gegenpol aufgebaut. Beide Gedichte reflektieren auch den politischen und literarischen Diskurs im Gefolge der Rheinkrise - ebenfalls in unterschiedlicher Weise. Nicht anders als Heine distanzierte sich auch Gottschall von Nikolaus Beckers populärem Gedicht Der deutsche Rhein und erteilt deshalb 1842 in seinem Gedicht Dem Rhein allen 'Franzosenfressern' eine Abfuhr - und dabei spielt auch die Loreley als Allegorie des Gesangs und der Dichtung eine positive Rolle:
Du, Vater Rhein, brauchst nicht der schönen Worte,
Du bist und bleibst der freie deutsche Rhein;
Einst sprengtest Du die enge Felsenpforte
In Jugendkraft, um freier noch zu sein.
Dann hüpftest jauchzend Du zum Meer hernieder;
Es sang die Loreley Dir ihre Lieder.
Und Du, der freie Sohn der freien Berge,
Den Poesie umduftet und umhaucht;
Du bist kein Gränzeswächter und kein Scherge,
Kein Knecht, der zum Beamtendienste taugt,
Der aufgepflanzt dasteht an Deutschlands Thoren,
Als wär' er uns vereidet und geschworen. (트)

1842 charakterisiert Gottschall den Rhein nicht als "Deutschlands Wächter", sondern als "Brücke", die "die Völker zu einander führt". Seine Loreley-Allegorie hat in diesem Zusammenhang nichts Dämonisches oder Krankes, sondern steht voll und ganz im Einklang mit dem Bild vom Rhein als völkerverbindender "Brücke". In dem etwa 40 Jahre später entstandenden Gedicht Am Rhein änderte sich diese Sicht wesentlich. Dieser Text führt nämlich die 'Hurra-patriotische' Rhein-Lyrik 
der 1840er Jahre weiter; doch ging es einem Nikolaus Becker nur um Grenzsicherung, so frohlockte Gottschall über die Annexion Elsaß-Lothringens. In Gottschalls Vers "Da steht sie mit dem Schwert auf der Wacht" klingt auch Max Schneckenburgers Gedicht Die Wacht am Rhein an; doch ging es Schneckenburger 1840 lediglich um eine Art 'Verteidigungsfall', so hat Gottschall ausschließlich die Sicherung der Siegesbeute von 1871 im Auge.

Dem mit Waffengewalt eingedeutschten Rhein gesteht Gottschall im Kaiserreich keine Brückenfunktion mehr zu; folgerichtig unterwirft er auch seine Loreley-Figur von 1842 einem Funktionswandel. Die frühere Entente cordiale zwischen Rhein und Loreley wird 1883 aufgegeben, weil der Rhein nun auch eine andere allegorische Gestalt am rechten Rheinufer hofiert: die Germania des Niederwald-Denkmals. Diese ist ausschließlich "Siegesgöttin" und hat mit der völkerverbindenden Freiheitsgöttin Germania, von der z.B. ein Ph. J. Siebenpfeiffer 1832 in seiner Rede auf dem Hambacher Fest spricht, (17) nur den Namen gemeinsam; die Hambacher Germania hat die Fackel der Aufklärung in der Hand, Gottschalls Germania dagegen Schwert und Kaiserkrone.

Der Literaturhistoriker Gottschall hatte Heines Loreley als "unvergänglich" gerühmt, das Gedicht aber trotzdem nur als Phänomen des Übergangs bewertet, - als Zeichen der Gesundung, nicht aber als Ausdruck der 'Gesundheit'. Auch der Dichter Gottschall dementiert in seinem Loreley/Germania-Gedicht von 1883 die Vorstellung von der Unvergänglichkeit der LoreleyAllegorie, wird sie doch als ein entnervtes Wesen am Rande der Selbstaufgabe dargestellt; zugleich deutet er hier aber auch an, daß der Untergang dieser Loreley zu verhindern ist, wenn sie sich auf ein Bündnis mit dem vaterländischen Vater Rhein und der Siegesgöttin einläßt. Gottschall demonstriert hier also einerseits die 'Vergänglichkeit' der bisherigen Loreley-Allegorie, zugleich versucht er aber auch, die Loreley-Symbolik zu aktualisieren und 'gesund' zu machen. Die 'Gesundheit', die bei Heine im wesentlichen "Postulat" bleibe, ist Gottschall zufolge greifbar nahe.

Meinte der Literaturhistoriker Gottschall, Heine habe vergessen, "daß nur diejenige Poesie, welche im Leben der Gegenwart wurzelt und von ihren Gedanken getragen wird, Dauer verspricht" (18), so versucht der Dicher Gottschall in seinem Loreley/Germania-Gedicht, die Loreley-Allegorie "im Leben der Gegenwart" der 1880er Jahre zu verwurzeln und seiner Vorstellung von Poesie auf diese Weise "Dauer" zu verleihen. Bei Heine hatte der Literaturhistoriker Gottschall "die ersten Keime der politischen Lyrik" gefunden, "welche aus dem mondhellen Zauberreich der Romantik wieder hinausführte in den sonnigen Tag der Geschichte." (모) Der Dichter Gottschall will diesen Weg nun fortsetzen und nimmt 1883 die als hinfällig dargestellte Loreley-Allegorie in die nationale Pflicht; doch er verzichtet auf Heine als Weggefährten, da

\section{[...] die Epoche der Heine'schen Poesie und ihrer maßgebenden Bedeutungen vorüber sei. Denn die auflösenden und zersetzenden Elemente sind einem künstlerischen Schöpfungsdrange gewichen, der sich nach ganzen Kunstwerken sehnt, der das moderne Leben in Gestalten aus Fleisch und Blut und zu ästhetischer Harmonie durchzubilden sucht. (므)}

Um dieses "moderne Leben" geht es Gottschall in seinem späten Loreley/Germania-Gedicht, denn er fordert die Schriftsteller darin implizit auf, sich dem neuen Deutschen Reich nicht zu verweigern. Aber er tut dies bezeichnenderweise nicht in "Gestalten aus Fleisch und Blut", sondern unter Berufung auf eine Germania, die im Text gleich dreimal als "Erzbild" in Erscheinung tritt.

Die Hoffnung des Literaturhistorikers Gottschall auf Ganzheit, Gesundheit, Geschlossenheit in Poesie und Politik und auf "organisches" Schaffen ist alles andere als "modern" oder fortschrittlich denn damit und mit seinem Loreley/Germania-Gedicht leistet er selbst der Errichtung eines "Zauberreichs der Romantik" Vorschub: und dies ist das neue Kaiserreich der National-Romantik. Gottschalls Germania ist somit nichts anderes als die martialische Neuinszenierung gerade jener abgetakelten Barbarossa- und Kyffhäuser-Romantik, die Heine im Wintermährchen verspottet hatte. Als Literaturkritiker hatte Gottschall Heines Versepos überaus positiv und nuanciert gewürdigt, (21) doch als Dichter und Deutscher meinte er, daraus keine Lehren ziehen zu müssen; in einer Zeit, als Deutschland vom eisernen Kanzler "durch Blut und Eisen" geeinigt worden war, behauptet Gottschall allen Ernstes, daß Heines Satire, "namentlich wo sie sich gegen das 
Preußenthum richtet, von den Ereignissen überholt worden ist." (222) - Auch in Heines Wintermährchen findet sich der Traum vom guten Kaiser und vom runderneuerten Reich, doch Heine zeigt zugleich, daß der Traum ein Ammenmärchen ist (DHA IV, 123). In diesem Kontext findet sich bei Heine auch ein positiv besetztes Germania-Bild, - Heine bedauert es nämlich, daß "Die theure, wundersame,/Goldlockigte Jungfrau Germania" (ebd.) einst "gemeuchelt" worden sei doch er denkt nicht im Traum daran, dieser Toten mit Hilfe der Literatur neues Leben einzuhauchen.

Die Loreley ist für Gottschall in mancher Hinsicht der Dreh- und Angelpunkt seiner Beurteilung des Dichters Heine, doch ähnliches kann man sagen über die Bedeutung der Loreley-Thematik für Gottschalls Selbstverständnis als Dichter, Denker und Deutscher. Das Loreley/Germania-Gedicht von 1883 will die frohe Botschaft vermitteln, daß mit der Errichtung des Kaiserreichs alle politische und romantische Zerrissenheit anachronistisch sei und daß auf die Poesie nun neue Aufgaben warten. Hatte Gottschall Heines Loreley als Verbündete im Kampf gegen die Romantik gesehen, so setzt er die Loreley-Allegorie im Loreley/Germania-Gedicht ironischerweise als Verbündete bei der Errichtung eines "Zauberreichs der nationalen Romantik" ein. Gottschalls Loreley/GermaniaGedicht ist Ausdruck der generellen Politisierung des Loreley-Motivs in Deutschland seit 1871. Als der Rhein nämlich aufhörte, ein französisch-deutscher Grenzfluss zu sein, mutierte die Loreley als "Allegorie des Gesangs und der Romantik" häufig zum "Symbol des Deutschtums schlechthin"; Edda Ziegler hat diese Entwicklung, die beinahe sogar dazu geführt hätte, daß die Germania-Statue des Niederwald-Denkmals auf dem Loreley-Felsen errichtet worden wäre, (23) zurecht als "nationalistische Pervertierung" bezeichnet. (24) Diese kulminiert in Siegbert Meyers Gedicht Die neue Loreley, wo die "deutscheste Jungfrau" dafür sorgt, daß "des Rheines Wellen" die Franzosen "verschlingen". (르)

Gottschall annuliert im Loreley/Germania-Gedicht sein frühes Gedicht von 1842, doch anders als bei Siegbert Meyer verschmelzen bei ihm Loreley und Germania nicht zu einer Figur. Er benötigt den Fortbestand der beiden allegorischen Gestalten, um zwei Pole zu haben, die er miteinander versöhnen und verbünden kann: "Wort" und "Tat", Geist und Macht, Poesie und Politik, Leier und Schwert, Lust- und Realitätsprinzip.

Humanistische Dichtung und teutonisches Säbelrasseln vertragen sich nicht! - Oder doch? Wir Nachgeborenen wissen, daß sich in der von Gottschall gewünschten friedlichen Koexistenz von Loreley und Germania die mörderische Koexistenz von Weimar und Buchenwald ankündigt. Und wir ahnen, daß der Horst-Wessel-Vers "Die Fahne hoch! Die Reihen dich geschlossen!" ein Zitat aus Gottschalls Loreley/Germania-Gedicht sein könnte, denn "die Reihen", die von Gottschalls Germania angeführt werden, sind bereits "fest geschlossen".

\section{Anmerkungen}

(1) Rudolf von Gottschall: Aus meiner Jugend. Erinnerungen. Berlin 1898, S. 363. «巛

(2) Vgl. hierzu Ernst-Ullrich Pinkert: "Du singst wie einst Tyrtäus sang." - Rudolf Gottschalls Gedicht Heine in den Fassungen von 1842 und 1849. - In: HJb 1990, S. 175-183. «

(3) Rudolf von Gottschall: Bunte Blüthen. Breslau u. Leipzig 1891, S. 48ff. u. 53f. «巛

(4) Deutsche Lyrik des neunzehnten Jahrhunderts bis zur modernen Ära. Mit einer literargeschichtlichen Einleitung, hrsg. von Rudolf von Gottschall. Leipzig [1908 oder 1909], S. 11. «巛

(5) [Rudolf Gottschall]: Lieder der Gegenwart. Königsberg 1842, S. 87-93. «巛

(6) [Anm. 4] - Gottschall hat hier von Heine 13 Gedichte abgedruckt, mehr als von jedem anderen deutschen Lyriker - allen voran die Loreley, S. 120f. «"

(7) Loreley in Kursivschrift verweist ausschließlich auf Heines Gedicht, Loreley in regulärer Schrifttype bezieht sich auf die allegorische Figur. ««

(8) Vgl. Rudolf Gottschall: Poetik. Die Dichtkunst und ihre Technik. Vom Standpunkt der Neuzeit. Breslau 1858, S. 138. «巛

(9) Rudolf Gottschall: Heinrich Heine's Entwickelungsgang nach neuen Quellen. - In: Unsere Zeit. Deutsche Revue der Gegenwart. Neue Folge 4. Leipzig 1868, S. 320-353; hier S. 336. «巛 
(10) Die deutsche Nationalliteratur des neunzehnten Jahrhunderts. Literarhistorisch und kritisch dargestellt von Rudolf Gottschall. Dritte vermehrte und verbesserte Auflage. Breslau 1872, Bd. 2, S. 65f. - Von diesem Buch erschienen bis 1881 fünf Auflagen. «巛

(11) Gottschall [Anm. 9], S. 62f. «巛

(12) Gottschall [Anm. 10], S. 62f. «巛

(13) Gottschall [Anm. 5], S. 60-64. «

(14) Gottschall [Anm. 3], S. 7-9. Vermutlich ist das Gedicht 1883 entstanden, als das im Text an zentraler Stelle erwähnte Niederwald-Denkmal bei Rüdesheim am Rhein eingeweiht wurde. «巛

(15) Vgl. Edda Zieglers Charakterisierung der Loreley als "Allegorie des Gesangs und der Romantik" (Edda Ziegler: Heinrich Heine. Leben - Werk - Wirkung. Zürich 1993, S. 225). «巛

(16) Gottschall [Anm. 5-], S. 62f. - Insgesamt 14 Strophen, hier Str. 9 und 10. «巛

(17) Vgl. Karl-Heinz Fingerhut (Hrsg.): Heine Heine: Deutschland. Ein Wintermärchen. Mit ergänzenden Texten zum historischen Verständnis engagierter Poesie des deutschen Vormärz. Frankfurt am Main u.a. 1976, S. 91. «

(18) Gottschall [Anm. 10], S. 77. «

(19) Gottschall [Anm. 9], S. 423. «

(20) Gottschall [Anm. 10], S. 80. «

(21) Gottschall [Anm. 10], S. 72f. «巛

(22) Gottschall [Anm. 9], S. 347. «巛

(23) Als 1871 nach einem Ort am Rhein gesucht wurde, wo man ein großes Nationaldenkmal errichten könnte, wurde vorübergehend - d.h. bevor man sich für den Niederwald bei Rüdesheim als Standort entschied - auch der Loreley-Felsen in Erwägung gezogen. Graf zu Eulenburg stellt am 16.11.1871 in Berlin diesbezüglich fest: "[...] der Niederwald möge festgehalten werden, die dagegen in Vorschlag gebrachte Lurley sei unzugänglich, unwirtlich und zu hoch." - Zit. in: Lutz Tittel: Das Niederwalddenkmal 1871-1883. Hildesheim 1979, S. 125. «

(24) Ziegler [Anm. 15], S. 225. «

(25) Siegbert Meyer: Die neue Loreley (um 1875). - Zit. in: Ziegler [Anm. 15], S. 227. «巛

Rudolf Gottschall: Am Rhein - Entstanden um 1883, veröffentlicht in Gottschall [Anm. 3], S. 7-9; hier wiedergegeben nach Gottschall [Anm. 4], S. 321-323:

Rudolf Gottschall: Am Rhein

Es hat der rebenumkränzte Rhein

Sein silbernes Haupt erhoben;

Er plaudert so gern im Mondenschein

Mit der stolzen Jungfrau droben.

Da steht sie mit dem Schwert auf der Wacht

Hoch auf des Berges Rücken,

Und alle Sterne der ewigen Nacht,

Sie scheinen ihr Haupt zu schmücken.

»O ragen wirst du zum Himmelsdom

Auf dem granitnen Kothurne,

Solang` ich gieße den mächtigen Strom

Aus unerschöpfter Urne.

Du hast mir ein langverlorenes Land

In meinen Kranz gewunden;

Es hat die Krone in deiner Hand

Den würdigen Keiser gefunden.

An deinem Sockel regt sich,

Als ob ein Nebel walle;

Ein Geisterschwarm umflutet dich,

Die Fürsten und Völker alle.

Da löst sich's von den Bildern los,

Die Reihen sind fest geschlossen: 
Du aber führst sie riesengroß,

Siegesgöttin, in Erz gegossen!

Du schützest den friedlichen Besitz

Und alle Friedenskronen;

Doch naht der Feind, dann schmettert dein Blitz

In seinen Legionen.

Ich grüße dich, Germania,

Auf deiner Bergeshalde!

Fest steht das Reich wie du selber da,

Erzbild vom Niederwalde.«

Und rastlos weiter rollt jetzt der Rhein

Durch Klippen die strudelnden Wellen,

Vorbei am ragenden Felsgestein,

An dem sie schäumend zerschellen.

Da steht auf der Höh' ein blasses Weib,

Verhüllt in graue Schleier,

So schattenhaft der schöne Leib,

Klanglos in der Hand die Leier.

Sie wird von flatterndem Gewand

Wie von dunklem Gewölk umflogen;

Sie neigt sich herab vom Felsenrand,

Bereit zum Sprung in die Wogen.

Der Locken Gold so erbleichend und matt,

Vom silbernen Gram durchwoben:

Die Leier vorauszuschleudern hat

Sie hoch die Hand erhoben.

Da ruft der Rhein ihr ein mächtiges Halt

Aus grollender Flut entgegen:

»Verzweifle nicht an der Lieder Gewalt,

Die dir im Herzen sich regen!

Oh neidlos sieh auf das erz'ne Weib,

Das sie begeistert feiern!

Noch tausende lockt dein süßer Leib,

In traumhaft wehenden Schleiern.

Noch tausende wird dein holdes Wort

Mit stiller Gunst beglücken:

Die Herzen reißt es im Taumel fort,

In seligem Entzücken.

Solang'ich vorbei an Burg und Dom

Und Rebenhügeln gleite,

Rauscht auch des deutschen Gesanges Strom

Gewaltig mir zur Seite.

O singe das Lied, das mutig und frei

Von Herz zu Herzen wandre!

Dein ist das Wort, o Lorelei;

Doch sprachlos ist die andre:

Die Göttin der Tat, so stolz und kühn

Blickt sie auf die Lande nieder;

Doch neben deutschen Taten blühn

Ja ewig die deutschen Lieder!« 
In: Ich Narr des Glücks. Heinrich Heine 1797 - 1856. Bilder einer Ausstellung. Hg. v. Joseph A. Kruse, Stuttgart / Weimar 1997, S. 435-441.

(c) Ernst-Ulrich Pinkert.

[Zurück]

Projekt Loreley, 2000 